\title{
VOLTERRA BOUNDARY CONTROL LAWS \\ FOR A CLASS OF NONLINEAR PARABOLIC PARTIAL DIFFERENTIAL EQUATIONS
}

\author{
Rafael Vazquez and Miroslav Krstic* \\ * Dept. of Mechanical and Aerospace Engineering, \\ University of California, San Diego, 9500 Gilman Drive, \\ La Jolla, CA 92093-0411, U.S.A.
}

\begin{abstract}
The efforts on boundary control of general classes of nonlinear parabolic PDEs with nonlinearities of superlinear growth have so far only resulted in counterexamples - results that show that finite time blow up occurs for large initial conditions even for simple cases like quadratic nonlinearities, with or without control. In this paper we present results identifying a class of systems that is stabilizable. Our approach is a direct infinite dimensional extension of the feedback linearization/backstepping approach and employs Volterra series nonlinear operators both in the transformation to a stable linear PDE and in the feedback law. While the full detail of our general approach is left for a future publication without a page limit, in this paper we give an example with explicit solutions for the plant/controller pair, including an explicit construction of the inverse of the feedback linearizing Volterra transformation. This, in turn, allows us to explicitly construct the exponentially decaying closed loop solutions. We include also a numerical illustration, showing blow up in open loop, and stabilization for large initial conditions in closed loop. Copyright (c)2004 IFAC
\end{abstract}

Keywords: Stabilization, Nonlinear control, Partial differential equations, Lyapunov Function, Boundary Conditions.

\section{INTRODUCTION}

Boundary control of linear parabolic PDEs is a well established subject with extensive literature, see (Lasiecka and Triggianii, 2000) and the references therein. On the other hand, boundary control of nonlinear parabolic PDEs is a completely open problem as far as general classes of systems are concerned. Applications of interest include fluids, thermal, chemically-reacting, and plasma systems. The book (Christofides, 2001) solves problems of nonlinear parabolic PDE control but for inside-the-domain actuation, rather than with boundary control.
When attempting to develop general methods for nonlinear PDEs, it is advisable to take a clue from finite dimensional nonlinear systems. Clearly, one should bet on methods that have emerged as successful there. This eliminates (direct) optimal control methods and leaves feedback linearization/backstepping/Lyapunov approaches as candidates for extension to PDEs. This implies that the optimal control/operator Riccati equation solutions (Lasiecka and Triggianii, 2000) are unlikely to be extended to the nonlinear case.

The backstepping approach for linear PDEs has reached the level of maturity where a systematic design procedure (Smyshlyaev and Krstic, 2003) is available for a broad general class of parabolic 
equations. This systematic procedure provides explicit formulae for control laws in most cases of practical interest and is the starting point for our nonlinear developments here.

Our efforts in this paper are based on Volterra series. This is a major departure from our previous discretization-based nonlinear efforts (Boskovic and Krstic, 2001; Boskovic and Krstic, 2002; Boskovic and Krstic, 2003; Aamo and Krstic, 2004), which were successful in addressing some applications but in general cannot be expected to converge when the discretization step goes to zero, as shown in (Balogh and Krstic, 2003).

We start the paper by reviewing the literature on blow up phenomena in nonlinear PDEs (Section 2), including results that show that blow up cannot be overcome by feedback in some physically relevant classes of parabolic PDEs. In Section 3 we introduce the general class of nonlinear parabolic PDEs we consider in this paper, where the nonlinearity is of the form of a nonlinear operator represented by a Volterra series whose kernels are functions of the spatial variables of the PDE. Section 4 is the main result of the paper, introducing a feedback linearization/backstepping type of an approach to stabilizing the PDE. A coordinate transformation (nonlinear and infinite dimensional, represented by a Volterra operator) is introduced to transform the original nonlinear PDE into a stable linear PDE (a heat equation, to be exact). Finding the kernels of the transformation is the main design task in this procedure. The full detail of the general design procedure is beyond the scope of this paper (due to the page limit), thus, in Section 5 we show an example where a simple, yet nonlinear, controller stabilizes a nonlinear PDE that has a finite time blow up in open loop, due to a superlinear nonlinearity in its model. As in feedback linearization and backstepping, the invertibility of the coordinate transformation to a linear system is the key question, which we address in Section 6, developing explicit expressions for the inverse transformation, and even providing explicit closed loop solutions for the PDE.

\section{BLOW UP PHENOMENA IN NONLINEAR PARABOLIC EQUATIONS}

One of the facts that has perhaps discouraged the boundary control community from attempting anything systematic in global nonlinear stabilization is that some of the basic nonlinear PDEs are not globally stabilizable. When considering parabolic partial differential equations of the kind

$$
u_{t}=u_{x x}+f(u),
$$

in the interval $(0,1)$, where $f(u)$ is a continuous (or even smooth) function, with, for example,
Dirichlet boundary conditions, finite-time blow up instabilities are likely to occur when $f(u)$ is superlinear. This was first studied in a classical paper (Fujita, 1966), and has been object of systematic study in subsequent years. More recent reviews include (Levine, 1990) and (Deng and Levine, 2000). Exponentially growing functions, which also lead to blow up, have been considered (Gelfand, 1963; Bebernes and Eberly, 1989) because their importance in some combustion problems. The analysis leading to blow up results usually makes use of differential inequalities and the maximum principle.

In recent years, the question of controllability of this kind of equations has been considered. For superlinear functions which grow faster than $|u| \log ^{p}(1+|u|)$ lack of controllability is proved in (Fernandez-Cara and Zuazua, 2000), along with a study of controllability depending on the value of the exponent $p$. Therefore, for many nonlinearities there are initial conditions which cannot be brought to zero, no matter what amount of control is applied. In (Coron and Trelat, 2003) a more hopeful result was shown, where the problem of moving between different steady states by means of boundary control is considered for nonlinearities with superlinear growth.

The negative results like these indicate that the class of systems to be controlled needs to be refined. If such a refinement is made at the very short length scales at which the blow ups occur, the refinement would not be non-physical since the PDEs don't model physics (fluids, materials) at the shortest length scales.

\section{CLASS OF SYSTEMS UNDER STUDY}

We study the following class of parabolic systems,

$$
u_{t}=u_{x x}+F[u]
$$

with $x \in(0,1)$, Dirichlet boundary conditions $u(0, t)=0$ and $u(1, t)=U(t)$, and initial condition $u(x, 0)=u_{0}(x)$, where $U(t)$ is the control input and $F[u]$ is a Volterra nonlinearity. A Volterra series is defined as

$$
F[u](x, t)=\sum_{i=1}^{\infty} F_{i}[u](x, t),
$$

where the notation $F_{i}[u](x, t)$ emphasizes the fact that each $F_{i}[u](x, t)$ is a functional of $u(x, t)$ and that it depends on $x$ and $t$. The precise definition of the terms, employing the triangular formulation of the kernels, is: 


$$
\begin{aligned}
F_{i}[u](x, t)= & \int_{0}^{x} \int_{0}^{\sigma_{1}} \ldots \int_{0}^{\sigma_{i-1}} f_{i}\left(x, \sigma_{1}, \ldots, \sigma_{i}\right) \\
& \times\left(\prod_{j=1}^{i} u\left(\sigma_{j}, t\right)\right) d \sigma_{1} \ldots d \sigma_{n}
\end{aligned}
$$

where $f_{i}$ is called the $i$ th Volterra (triangular) kernel.

Volterra series (Volterra, 1959) are widely known and studied in the control literature (Boyd et al., 1984; Isidori, 1995; Lamnabhi-Lagarrigue, 1996; Sastry, 1999). An excellent exposition can also be found in (Rugh, 1981).

\section{CONTROL STRATEGY}

The objective is to find a stabilizing Volterra feedback law for $U(t)$. As in the feedback linearization approach for finite dimensional systems, we transform the original plant into another system, which we call target system. As target system we choose the simplest possible PDE with the properties we need, but also similar enough in structure to the original system. The natural choice for this problem is the heat equation

$$
w_{t}=w_{x x}
$$

with homogeneous boundary conditions $w(0, t)=$ $w(1, t)=0$, which is exponentially stable by standard linear PDE results.

To transform (2) into (5), a change of variables is used which is based again on a Volterra series:

$$
\begin{aligned}
w(x, t) & =u(x, t)-K[u](x, t) \\
& =u(x, t)-\sum_{i=1}^{\infty} K_{i}[u](x, t) .
\end{aligned}
$$

Evaluating (6) at $x=1$ and using the boundary conditions of both the original and the target system, we arrive at the control law:

$$
U(t)=\sum_{i=1}^{\infty} K_{i}[u](1, t)
$$

Assuming that the series kernels can be differentiated, using (2) and (5) the following equation is obtained:

$$
\sum_{i=1}^{\infty} F_{i}[u]=\sum_{i=1}^{\infty}\left(\frac{\partial}{\partial t} K_{i}[u]-\frac{\partial^{2}}{\partial x^{2}} K_{i}[u]\right) .
$$

Using (8), the definition of each term in the Volterra series, integration by parts, and change of the order of integration in the multiple integrals that arise, we obtain a set of partial differential equations for the kernels $k_{i}$ of $K_{i}$.

\section{AN EXAMPLE OF A STABILIZABLE SUPER-LINEAR SYSTEM}

For illustration we present a plant/controller pair with superlinear nonlinearities (and open loop blow up) where the controller achieves stabilization. The pair is chosen such that the controller is as simple as possible so the design concept is clear without getting lost in the technicalities.

The simplest possible (nonlinear) controller comes from a single second order control kernel, $k_{2}=$ $\sigma_{1} \sigma_{2}\left(x-\sigma_{1}\right)\left(x-\sigma_{2}\right)$, whose particular form comes from (8). All other control kernels are set to zero, i.e., $k_{1}=k_{3}=\ldots=k_{n}=\ldots=0$. Then the control input, $U(t)=K[u](1, t)$, is:

$$
\begin{aligned}
U(t)= & \int_{0}^{1} \int_{0}^{\sigma_{1}} \sigma_{1} \sigma_{2}\left(1-\sigma_{1}\right)\left(1-\sigma_{2}\right) \\
& \times u\left(\sigma_{1}, t\right) u\left(\sigma_{2}, t\right) d \sigma_{1} d \sigma_{2},
\end{aligned}
$$

which can be written shorter thanks to the symmetry of the kernel:

$$
U(t)=\frac{1}{2}\left(\int_{0}^{1} \sigma(1-\sigma) u(\sigma, t) d \sigma\right)^{2}
$$

The plant kernels derived from $(8)$ are $f_{1}=0$,

$$
\begin{aligned}
f_{2}= & 2 \sigma_{2} \sigma_{1}+2 \sigma_{2} x-2 \sigma_{2}^{2}+2 \sigma_{1} x-2 \sigma_{1}^{2}, \\
f_{3}= & \sigma_{1} \sigma_{2} x\left(\frac{x \sigma_{2}}{3}\left(\sigma_{2}^{2}+\sigma_{1} x\right)-\frac{\sigma_{2}^{4}}{6}-\frac{\sigma_{1}^{4}}{6}\right. \\
& -\frac{x^{3}}{2}\left(\sigma_{1}+\sigma_{2}\right)+\frac{\sigma_{1}^{3}}{3}\left(x-\sigma_{2}\right)+\frac{\sigma_{2} \sigma_{1}}{3}\left(x^{2}-\sigma_{2}^{2}\right) \\
& \left.+\frac{x^{4}}{3}\right)+\sigma_{1} \sigma_{3} x\left(\frac{x^{4}}{3}+\frac{\sigma_{1}^{3}}{3}\left(x-\sigma_{3}\right)+\frac{2 \sigma_{3} \sigma_{1} x^{2}}{3}\right. \\
& \left.-\frac{\sigma_{1}^{4}}{6}-\frac{x^{3}}{2}\left(\sigma_{1}+\sigma_{3}\right)\right)+\sigma_{2} \sigma_{3} x^{2}\left(\frac{x^{2}}{3}\right. \\
& \left.-\frac{x}{2}\left(\sigma_{2}-\sigma_{3}\right)+\frac{2 \sigma_{2} \sigma_{3}}{3}\right)+\frac{\sigma_{1}^{2} \sigma_{2} \sigma_{3}}{6} \\
& \times\left(4 \sigma_{3} \sigma_{2}\left(2 \sigma_{1}+\sigma_{2}\right)-7 \sigma_{2}^{3}-7 \sigma_{1}^{2}\left(\sigma_{2}+\sigma_{3}\right)\right) \\
& +\frac{\sigma_{1}^{2}}{6}\left(\sigma_{2}^{5}+\sigma_{1}^{3}\left(\sigma_{3}^{2}+\sigma_{2}^{2}\right)\right)+\frac{x \sigma_{1} \sigma_{2} \sigma_{3}}{6}\left(6 x \sigma_{3} \sigma_{1}\right. \\
& +7 \sigma_{2}^{3}+14 \sigma_{1}^{3}+\sigma_{2}^{2}\left(10 \sigma_{1}-4 \sigma_{3}-10 x\right) \\
& -20 \sigma_{1}^{2} x+6 x \sigma_{3} \sigma_{2}+2 x^{2}\left(3 x-\sigma_{1}-\sigma_{2}-\sigma_{3}\right) \\
& \left.+6 \sigma_{1}\left(\sigma_{3} \sigma_{1}+\sigma_{2} \sigma_{1}+x \sigma_{2}-3 \sigma_{2} \sigma_{3}\right)\right), \\
f_{n}= & \int_{\sigma_{2}}^{\sigma_{1}} k_{2}\left(x, \sigma_{1}, s\right) f_{n-1}\left(s, \sigma_{2}, \ldots, \sigma_{n}\right) d s \\
+ & \sum_{\gamma} \widehat{P}_{n}(\bar{\sigma}) \\
& \times f_{n-1}\left(s, \gamma_{2}, \ldots, \gamma_{n}\right) d s,
\end{aligned}
$$

where $\bar{\gamma}=\left(\gamma_{1}, \ldots, \gamma_{n}\right), \bar{\sigma}=\left(\sigma_{1}, \ldots, \sigma_{n}\right)$, and $\widehat{P_{n}}\left(\sigma_{1}, \ldots, \sigma_{n}\right)$ is a certain subset of the permutations of the variables $\left(\sigma_{1}, \ldots, \sigma_{n}\right)$, used to write 


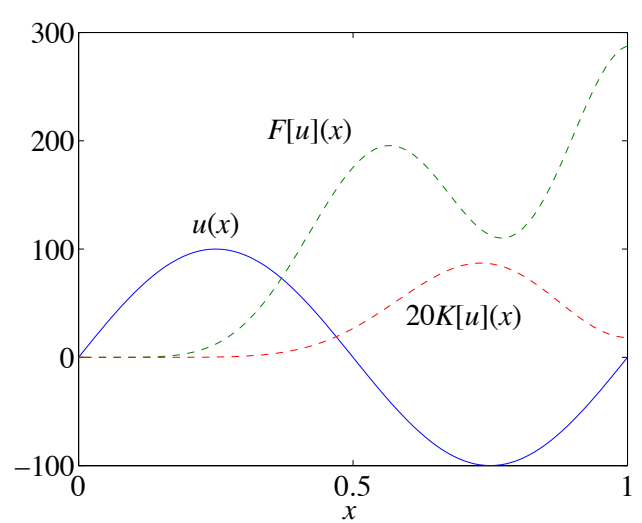

Fig. 1. Effect of $K$ and $F$ on $u(x)=100 \sin (2 \pi x)$ the equations in compact form, and defined in the following fashion:

Definition 1. Given an ordered set of $n$ different objects, say $\left(\xi_{1}, \ldots, \xi_{n}\right)$, we define $\widehat{P_{n}}\left(\xi_{1}, \ldots, \xi_{n}\right)$ as the set of $n$ ordered $n$-tuples, $\left\{S_{1}, \ldots, S_{n}\right\}$ which are given by the following recursive formula:

$$
\begin{aligned}
S_{1} & =\left(\xi_{1}, \ldots, \xi_{n}\right), \\
S_{i} & =s_{1, i} S_{i-1}, \quad i=2, \ldots, n,
\end{aligned}
$$

where $s_{1, i}$ denotes the operation of flipping between the first and the $i$ th element.

For example,

$$
\begin{aligned}
\widehat{P_{4}}(1,2,3,4)=\{ & (1,2,3,4),(2,1,3,4), \\
& (3,1,2,4),(4,1,2,3)\} .
\end{aligned}
$$

Using this definition and employing symbolic calculation it is easy to get all the kernels up to a desired order. Higher order kernels get smaller and smaller, and its influence becomes negligible.

For the purpose of illustrating the operators $K$ and $F$, we plot the effect of both them on a test function, $u(x)=100 \sin (2 \pi x)$ in Figure 1 . The order of magnitude of $K$ is much less than the order of magnitude of $F$, so we plot $20 K$ for the sake of clarity.

Starting with a large enough initial condition (of the order of 200), the uncontrolled system diverges to infinity in finite time, as seen in Figure 2. With the controller (9), this behavior is suppressed and the system is stabilized, as shown in Figure 2.

\section{INVERSE OF THE CHANGE OF VARIABLES, STABILITY, AND EXPLICIT CLOSED LOOP SOLUTIONS}

For proving stability of the controlled system in the example, the first step is to show the invertibility of the change of variables
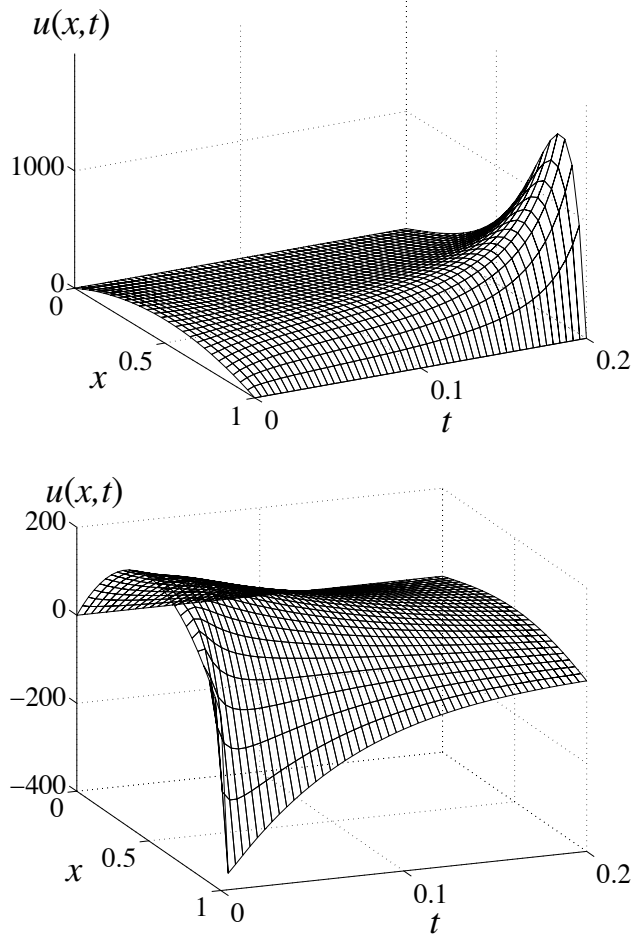

Fig. 2. Uncontrolled and controlled system.

$$
\begin{aligned}
w(x, t)= & u(x, t)-\int_{0}^{x} \int_{0}^{\sigma_{1}} \sigma_{1} \sigma_{2}\left(x-\sigma_{1}\right)\left(x-\sigma_{2}\right) \\
& \times u\left(\sigma_{1}, t\right) u\left(\sigma_{2}, t\right) d \sigma_{2} d \sigma_{1} .
\end{aligned}
$$

It is natural to also seek a Volterra formulation for the inverse $u=w+L[w]$, i.e.,

$$
\begin{aligned}
u(x, t)= & w(x, t)+\int_{0}^{x} \int_{0}^{\sigma_{1}} \ldots \int_{0}^{\sigma_{i-1}} l_{i}\left(x, \sigma_{1}, \ldots, \sigma_{i}\right) \\
& \times\left(\prod_{j=1}^{i} w\left(\sigma_{j}, t\right)\right) d \sigma_{1} \ldots d \sigma_{n} .
\end{aligned}
$$

To find the Volterra kernels $l_{i}$, substituting (6) into (18), we arrive at

$$
K=L-L K,
$$

where, in our example, $K=K_{2}$. Expanding the Volterra series $L$ and matching the terms, a set of recursive linear integral equations for the $l_{i}$ 's can be found for the general case. For our example, the integral equations simplify to direct computation of several integrals, which can be obtained explicitly using symbolic software as $l_{1}=0$, 


$$
\begin{aligned}
l_{2}= & \sigma_{1} \sigma_{2}\left(x-\sigma_{1}\right)\left(x-\sigma_{2}\right), \\
l_{3}= & \sigma_{1} \sigma_{2} \sigma_{3}\left[( 2 x - \sigma _ { 2 } - \sigma _ { 3 } ) \left(\frac{\sigma_{1}^{5}-x^{5}}{5}\right.\right. \\
& \left.+\frac{x^{4}-\sigma_{1}^{4}}{4}\left(x+\sigma_{1}\right)+\frac{\sigma_{1}^{3}-x^{3}}{3} x \sigma_{1}\right) \\
& +\left(x\left(\sigma_{2}+\sigma_{3}\right)-\sigma_{2} \sigma_{3}\right)\left(\frac{x^{4}+\sigma_{1}^{4}}{4}\right. \\
& \left.+\frac{\sigma_{1}^{3}-x^{3}}{3}\left(x+\sigma_{1}\right)+\frac{x^{2}+\sigma_{1}^{2}}{2} x \sigma_{1}\right) \\
& +\left(x-\sigma_{1}\right)\left(\frac{\sigma_{2}^{5}-x^{5}}{5}+\frac{x^{4}+\sigma_{2}^{4}}{4}\right. \\
& \times\left(x+\sigma_{2}+\sigma_{3}\right)+\frac{\sigma_{2}^{3}-x^{3}}{3}\left(x\left(\sigma_{2}+\sigma_{3}\right)\right. \\
& \left.\left.\left.+\sigma_{2} \sigma_{3}\right)+\frac{x^{2}+\sigma_{2}^{2}}{2} x \sigma_{2} \sigma_{3}\right)\right],
\end{aligned}
$$

and so on.

The key question is whether the Volterra series $L$ converges. This question is answered using Theorem 3.3.1 on inverses of Volterra series in (Boyd et al., 1984). Applied to our problem, this theorem states that, if the operator Id $K_{1}$ is invertible then $\mathrm{Id}+L$ converges locally in the spatial $L_{\infty}$ sense, i.e., for sufficiently small $w(x, t)$. It was proved in (Smyshlyaev and Krstic, 2003) that backstepping guarantees Id $-K_{1}$ to be invertible, thus local invertibility is guaranteed in the general case. In our example $K_{1}=0$, thus the condition of Boyd's theorem is satisfied trivially, and hence $\operatorname{Id}+L$ converges for small $w(x, t)$.

With the $l_{i}$ 's obtained explicitly, we also obtain the explicit solution for $(5)$ in terms of $u_{0}(x)$ :

$$
\begin{aligned}
w(x, t)= & 2 \sum_{n=1}^{\infty} e^{-\pi^{2} n^{2} t} \sin (\pi n x) \\
& \times \int_{0}^{1} \sin (\pi n \xi)\left[u_{0}(\xi)\right. \\
& \left.-\frac{1}{2}\left(\int_{0}^{\xi} \eta(\xi-\eta) u_{0}(\eta) d \eta\right)^{2}\right] d \xi(22
\end{aligned}
$$

By substituting (22) into (18), with (20), (21) we obtain an explicit expression for $u(x, t)$. The resulting expression contains a Volterra series which converges for sufficiently small $u_{0}(x)$, establishing at least local exponential stability of the closed loop system. As the bottom in Figure 2 shows, the actual stability result is much more than local.

\section{CONCLUSIONS}

The efforts on nonlinear boundary control of PDEs of parabolic type have so far resulted primarily in negative results - results that show that control cannot prevent finite time blow up. In this paper we show the first positive result that stabilizes a PDE with a superlinear nonlinearity and open loop blow up. Our design is based on a general approach utilizing Volterra operators in the nonlinear feedback law, which we don't present in detail due to space constraints. This approach is the first rigorous, continuum approach to boundary control for general classes of nonlinear parabolic PDEs and, unlike previous efforts (including ours), does not employ any spatial discretization.

The approach we presented in the paper is a direct infinite dimensional extension of feedback linearization/backstepping. A PDE, with a Volterra nonlinearity, is transformed via an invertible coordinate transformation represented in the Volterra form, and via a nonlinear feeback law in the Volterra form, into the (linear, stable) heat equation.

In the example that we presented in the paper, every element of the design and analysis is explicit - the plant, the controller, the linearizing transformation, and even the closed loop solution. This may not always be possible for a general $F[u]$, however, if the linear case (Smyshlyaev and Krstic, 2003) is an indicator, it might be possible for a number of relevant subclasses.

While in this paper we postulated the control law for our example, in a general problem, finding the controller's Volterra kernels would be the main task. As we indicated in Section 4, to find the kernels $k_{i}$, a recursive set of linear hyperbolic PDEs on spaces of increasing dimensions and with moving boundaries would have to be solved, which will be presented in a future, longer paper. Future efforts include studying the well-posedness of these PDEs, their numerical solution, and the convergence of the resulting Volterra series $K[u]$.

While common physical PDE models mostly come with nonlinear functions $f(u(x, t))$, rather than with Volterra series $F[u](x, t)$, our approach can be adapted to be used in such cases. For example, if $f(u)=u^{2}$, we would approximate it with $F[u]=F_{2}[u]$ with

$$
f_{2}\left(x, \sigma_{1}, \sigma_{2}\right)=\eta\left(x-\sigma_{1}\right) \eta\left(x-\sigma_{2}\right),
$$

where (Evans, 1998)

$$
\eta(\xi)=\frac{c}{\epsilon} \exp \left(\frac{\epsilon^{2}}{\xi^{2}-\epsilon^{2}}\right)(h(\xi+\epsilon)-h(\xi-\epsilon))
$$

is a $C^{\infty}$ function, $h$ is the step function, $c=$ $\left(\int_{-1}^{1} \exp \left(\left(x^{2}-1\right)^{-1}\right) d x\right)^{-1}$, and $\epsilon$ controls the tightness of the approximation. Obviously, the blow up for $f(u)=u^{2}$ with large initial conditions cannot be prevented with any boundary controller. Thus we would expect our approximate controller for $F[u]=F_{2}[u]$, when applied 
to $f(u)=u^{2}$, to be effective only for initial data of limited size.

\section{REFERENCES}

Aamo, O.M. and M. Krstic (2004). Boundary control of the linearized Ginzburg-Landau model of vortex shedding. European Journal of Control. to appear.

Balogh, A. and M. Krstic (2003). Infinite dimensional backstepping for nonlinear parabolic PDE's. In: Sixty Open Problems in the Mathematics of Systems and Control (V. Blondel and A. Megretski, Eds.). Princeton University Press. Princeton, NJ.

Bebernes, J. and D. Eberly (1989). Mathematical Problems from Combustion Theory. SpringerVerlag. New York.

Boskovic, D. and M. Krstic (2001). Nonlinear stabilization of a thermal convection loop by state feedback. Automatica 37, 2033-2040.

Boskovic, D. and M. Krstic (2002). Backstepping control of chemical tubular reactors. Computers and Chemical Engineering 26, 1077-1085.

Boskovic, D. and M. Krstic (2003). Stabilization of a solid propellant rocket instability by state feedback. lnternational Journal of Robust and Nonlinear Control 13, 483-495.

Boyd, S., L.O. Chua and C.A. Desoer (1984). Analytical foundations of Volterra series. Journal of Mathematical Control and Information 1, 243-282.

Christofides, P.D. (2001). Nonlinear and Robust Control of Partial Differential Equation Systems: Methods and Applications to TransportReaction Processes. Birkhauser. Boston.

Coron, J.M. and E. Trelat (2003). Global steadystate controllability of $1-\mathrm{D}$ semilinear heat equation. Preprint.

Deng, K. and H.A. Levine (2000). The role of critical exponents in blow-up theorems: the sequel. Journal of Mathematical Analysis and Applications 243, 85-126.

Evans, L.C. (1998). Partial Differential Equations. American Mathematical Society. Providence, Rhode Island.

Fernandez-Cara, E. and E. Zuazua (2000). Null and approximate controllability for weakly blowing up semilinear heat equations. Annales de l'IHP. Analyse non Linéaire 17, 583-616.

Fujita, H. (1966). On the blowing up of solutions of the Cauchy problem for $u_{t}=\Delta u+u^{1+\alpha}$. Journal of the Faculty of Sciences, University of Tokyo, Section IA Mathematics 13, 105113.

Gelfand, I.M. (1963). Some problems in the theory of quasilinear equations. American Mathematical Society Translations 19, 295-381.
Isidori, A. (1995). Nonlinear Control Systems. Springer-Verlag. Berlin.

Lamnabhi-Lagarrigue, F. (1996). Volterra and Fliess series expansions for nonlinear systems. In: The Control Handbook (W.S. Levine, Ed.). pp. 879-888. CRC Press. Boca Raton, FL.

Lasiecka, I. and R. Triggianii (2000). Control Theory for Partial Differential Equations: Continuous and Approximation Theories. Vol. 1. Cambridge University Press. Cambridge, UK.

Levine, H.A. (1990). The role of critical exponents in blow-up theorems. SIAM Review 32, 262288.

Rugh, W.J (1981). Nonlinear Sytem Theory: The Volterra/Wiener Approach. Web version. www.ece.jhu.edu/ rugh/volterra/book.pdf.

Sastry, S. (1999). Nonlinear Systems: Analysis, Stability and Control. Springer-Verlag. New York.

Smyshlyaev, A. and M. Krstic (2003). Regularity of hyperbolic PDE's governing backstepping gain kernels for parabolic PDE's. In: Proc. of the 2003 ACC. Vol. 3. pp. 2634-2639.

Volterra, V. (1959). Theory Of Functionals And Of Integral And Integro-Differential Equations. Dover. New York. 